Kundennutzen durch digitale Transformation 
Elke Brucker-Kley

Denisa Kykalová

Thomas Keller

(Hrsg.)

\section{Kundennutzen \\ durch digitale \\ Transformation}

Business-Process-Management-Studie Status quo und Erfolgsmuster

算 Springer Open 
Herausgeber

Elke Brucker-Kley

Institut für Wirtschaftsinformatik

ZHAW School of Management and Law

Winterthur, Schweiz

Denisa Kykalová

Sanacare AG

Winterthur, Schweiz
Thomas Keller

Institut für Wirtschaftsinformatik

ZHAW School of Management and Law

Winterthur, Schweiz

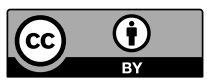

ISBN 978-3-662-55706-8 ISBN 978-3-662-55707-5 (eBook)

https://doi.org/10.1007/978-3-662-55707-5

Die Deutsche Nationalbibliothek verzeichnet diese Publikation in der Deutschen Nationalbibliografie; detaillierte bibliografische Daten sind im Internet über http://dnb.d-nb.de abrufbar.

\section{Springer Gabler}

Dieses Buch ist eine Open-Access-Publikation.

(C) Der/die Herausgeber bzw. der/die Autor(en) 2018

Open Access Dieses Buch wird unter der Creative Commons Namensnennung 4.0 International Lizenz (http:// creativecommons.org/licenses/by/4.0/deed.de) veröffentlicht, welche die Nutzung, Vervielfältigung, Bearbeitung, Verbreitung und Wiedergabe in jeglichem Medium und Format erlaubt, sofern Sie den/die ursprünglichen Autor(en) und die Quelle ordnungsgemäß nennen, einen Link zur Creative Commons Lizenz beifügen und angeben, ob Änderungen vorgenommen wurden.

Die in diesem Buch enthaltenen Bilder und sonstiges Drittmaterial unterliegen ebenfalls der genannten Creative Commons Lizenz, sofern sich aus der Abbildungslegende nichts anderes ergibt. Sofern das betreffende Material nicht unter der genannten Creative Commons Lizenz steht und die betreffende Handlung nicht nach gesetzlichen Vorschriften erlaubt ist, ist für die oben aufgeführten Weiterverwendungen des Materials die Einwilligung des jeweiligen Rechteinhabers einzuholen.

Die Wiedergabe von Gebrauchsnamen, Handelsnamen, Warenbezeichnungen usw. in diesem Werk berechtigt auch ohne besondere Kennzeichnung nicht zu der Annahme, dass solche Namen im Sinne der Warenzeichen- und Markenschutz-Gesetzgebung als frei zu betrachten wären und daher von jedermann benutzt werden dürften. Der Verlag, die Autoren und die Herausgeber gehen davon aus, dass die Angaben und Informationen in diesem Werk zum Zeitpunkt der Veröffentlichung vollständig und korrekt sind. Weder der Verlag noch die Autoren oder die Herausgeber übernehmen, ausdrücklich oder implizit, Gewähr für den Inhalt des Werkes, etwaige Fehler oder Äußerungen. Der Verlag bleibt im Hinblick auf geografische Zuordnungen und Gebietsbezeichnungen in veröffentlichten Karten und Institutionsadressen neutral.

Gedruckt auf säurefreiem und chlorfrei gebleichtem Papier

Springer Gabler ist ein Imprint der eingetragenen Gesellschaft Springer-Verlag GmbH, DE und ist ein Teil von Springer Nature.

Die Anschrift der Gesellschaft ist: Heidelberger Platz 3, 14197 Berlin, Germany 


\section{Danksagung}

Das Studienteam der ZHAW School of Management and Law dankt allen an den Fallstudien beteiligten Unternehmensvertretern, die ihre wertvolle Zeit für die Erarbeitung, Diskussion und Auswertung bereitgestellt haben. Auf der Grundlage ihrer Erfahrungen ist es gelungen, Herausforderungen, Lösungswege und Erfolgsmuster im Kontext der digitalen Transformation für ein breites Spektrum an Branchen und Anwendungsfällen zusammenzutragen und zu teilen.

Die Business-Process-Management-Studie und das BPM-Symposium an der ZHAW School of Management and Law sind Gefässe der angewandten Forschung und Entwicklung, die ohne die Beiträge von Wirtschaftspartnern nicht möglich sind. Das Studienteam der ZHAW School of Management and Law dankt den Unternehmen Appway, AMAG Leasing, Swisscom und Vontobel nicht nur für die finanzielle Unterstützung, sondern insbesondere für die Sicherstellung der Praxisrelevanz der Studie. 


\section{Herausgeber und Autoren}

\section{Die Praxisvertreter und Co-Autoren der Fallstudien}

Patrick Frauchiger, Leiter Marketing und Verkauf, AMAG Leasing AG, Baden-Dättwil

Esad Ceranic, Projektverantwortlicher \& Business Analyst, AMAG Leasing AG, BadenDättwil

Markus Schneider, Leiter Operation Services, Vontobel, Zürich

Philipp Klauser, Applikationsverantwortlicher Jira, Vontobel, Zürich

Andreas Hilber, Head of Process Architecture Network \& IT Operations, Swisscom AG, Zürich

Marco Peyer, Head BPM \& Service Operations, Swiss Re, P\&C Reinsurance, Adliswil

Lukas Steudler, Leiter Geschäftsstelle egovpartner.zh.ch, Staatskanzlei des Kantons Zürich, Zürich

\section{Die Herausgeber und das Autorenteam der Zürcher Hochschule für Angewandte Wissenschaften School of Management and Law, Winterthur}

Elke Brucker-Kley ist Leiterin des BPM Research Lab am Institut für Wirtschaftsinformatik der ZHAW School of Management and Law. Sie betreut studentische Arbeiten in Kooperation mit Wirtschaftspartnern und leitet Forschungs- und Beratungsprojekte in den Themenbereichen Solution Design, Prozessmanagement und Enterprise Architecture. Ihr Forschungsschwerpunkt liegt auf der Nutzung interdisziplinärer Ansätze zur Gestaltung wirksamer soziotechnischer Systeme.

Denisa Kykalová ist Fachspezialistin für medizinische Programme bei der Sanacare AG in Winterthur. Sie war zum Zeitpunkt der Studie wissenschaftliche Mitarbeiterin und Projektleiterin am Institut für Wirtschaftsinformatik der ZHAW School of Management and Law und leitete dort Forschungs- und Beratungsprojekte in den Themenbereichen Informations- und Prozessmanagement in verschiedensten Branchen mit einem Fokus auf dem Energiesektor.

Thomas Keller ist Professor am Institut für Wirtschaftsinformatik der ZHAW School of Management and Law. Er doziert zu den Themen Prozessautomatisierung und Enterprise Architecture im Bachelor- und Masterstudiengang Wirtschaftsinformatik und in der Weiterbildung. Zu seinen Forschungsschwerpunkten zählen Prozessautomatisierung, Prozessintegration und Enterprise Engineering. 
David Grünert ist wissenschaftlicher Mitarbeiter am Institut für Wirtschaftsinformatik der ZHAW School of Management and Law. Er unterrichtet Software Engineering im Studiengang Wirtschaftsinformatik und forscht zu neuen Modellierungsansätzen für Geschäftsprozesse sowie dem Einsatz von Gesten- und Sprachsteuerung zur Bearbeitung von Modellen.

Simon Näpflin ist wissenschaftlicher Assistent am Institut für Wirtschaftsinformatik der ZHAW School of Management and Law.

Sandro Graf ist Dozent am Institut für Marketing Management der ZHAW School of Management and Law und leitet die Fachstelle Service Lab. Er unterrichtet Konsumentenverhalten, Service Design und Customer Experience Management. Sein Team beschäftigt sich vorwiegend mit der Wahrnehmung von Konsumenten und unterstützt Unternehmen bei der Verbesserung der vermittelten Kundenerlebnisse.

Amélie-Charlotte Körner ist wissenschaftliche Mitarbeiterin am Institut für Marketing Management der ZHAW School of Management and Law. Neben ihrer Lehrtätigkeit leitet und unterstützt sie mit ihrem Hintergrund als Psychologin und qualitative Marktforscherin vor allem qualitative Forschungsprojekte. 


\section{Inhaltsverzeichnis}

\section{Studienrahmen}

$1 \quad$ Prozessmanagement als Gestaltungshebel der digitalen Transformation? . . 3 Elke Brucker-Kley, Thomas Keller und Denisa Kykalová

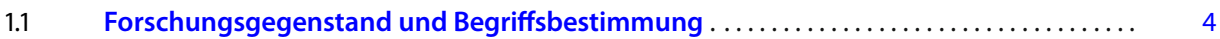

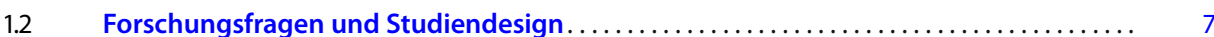

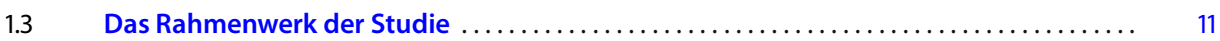
Literatur ....................................................... 16

\section{Die Quantitative Studie - Status quo}

2 Ergebnisse der Umfrage: «Status quo: Kundennutzen durch digitale Transformation?» ...............................

Denisa Kykalová, Elke Brucker-Kley und Simon Näpflin

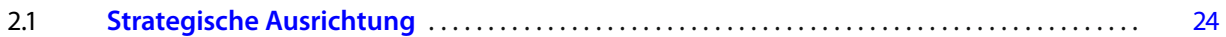

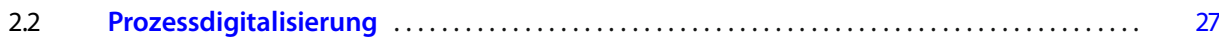

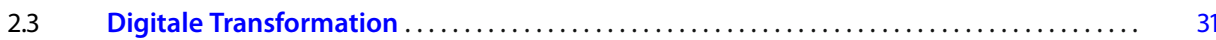

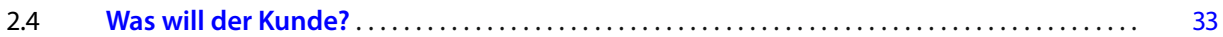

$2.5 \quad$ Kundenperspektive in der Prozessgestaltung

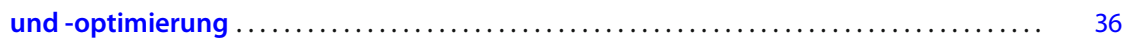

2.6 Kundendaten: Quellen, Nutzung und Einsatz für die Prozessausführung ........... 41

III Die Fallstudien - Erfolgsmuster aus der Praxis

3 «Erfolgsmuster: Kundennutzen durch digitale Transformation?»Einleitung zu den Fallstudien Elke Brucker-Kley

4 Fallstudie Amag Leasing AG: «Videobasierte Identifikation als Wegbereiter für das Online-Leasing".

Esad Ceranic, Patrick Frauchiger, Elke Brucker-Kley, Sandro Graf und Amélie-Charlotte Körner

4.1 Kontext und Ausgangssituation

Fazit

Literatur 
Literatur

Fallstudie Swiss Re: «Case Management in P\&C Reinsurance Wissensarbeit im Fokus»

Marco Peyer, David Grünert und Elke Brucker-Kley

Kontext und Ausgangssituation

Literatur

Fallstudie Vontobel: «Digitalisiertes Service Management schafft Kundennutzen»

Markus Schneider, Philipp Klauser und Thomas Keller

Kontext und Ausgangssituation .

Motivation und Zielsetzung

Fazit

Literatur

Fallstudie Swisscom: «Die Einführung von Produktionsund Servicekatalogen - Der erste Schritt zur industrialisierten Fertigung» .

Andreas Hilber, Denisa Kykalová und Elke Brucker-Kley

8.1 Kontext und Ausgangssituation

Fazit

Literatur

\section{Fazit und Ausblick}

9 Fazit: Prozessmanagement als Gestaltungshebel der digitalen

Transformation?

Elke Brucker-Kley, Thomas Keller und Denisa Kykalová

9.1 Status Quo «Kundennutzen durch Digitale Transformation»:

Fazit aus der Online-Befragung

Erfolgsmuster: «Kundennutzen durch Digitale Transformation» -

Fazit aus den Fallstudien

BPM Quo Vadis? - Prozessmanagement als Gestaltungshebel

der Digitalen Transformation 
Inhaltsverzeichnis

\section{Anhang}

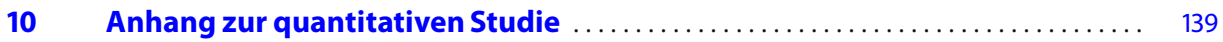

Denisa Kykalová

$10.1 \quad$ Teilnehmerkreis der Online-Befragung $\ldots \ldots \ldots \ldots \ldots \ldots \ldots \ldots \ldots \ldots \ldots \ldots \ldots \ldots \ldots$

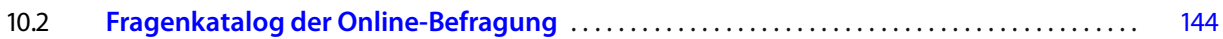




\section{Abbildungsverzeichnis}

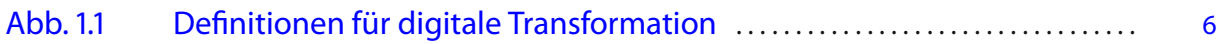

Abb.1.2 Forschungsfragen .......................................... 9

Abb. 1.3 Studiendesign und Ablauf .................................... 11

Abb. 1.4 Rahmenwerk der BPM Studie: Kundennutzen durch digitale Transformation? Gestaltungsfelder und Potenziale für das Prozessmanagement $\quad 12$

Abb.1.5 Gestaltungsziele der digitale Transformation - Wozu und für Wen? .... 14

Abb. 1.6 Gestaltungsfelder der Digitalen Transformation - Was wird transformiert? ....

Abb. 1.7 Digitale Transformation - Wie und Womit? (Formen der Prozessdigita-

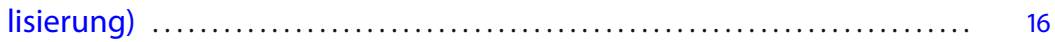

Abb. $2.1 \quad$ Unterforschungsfragen für die 6 Themenbereiche $\ldots \ldots \ldots \ldots \ldots \ldots \ldots . \ldots 22$

Abb. 2.2 Teilnehmende nach Grösse des Unternehmens (KMU vs. Grosse Unter-

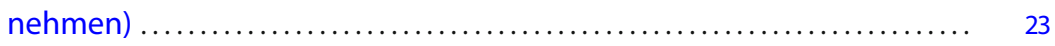

Abb. $2.3 \quad$ Strategische Ziele des Prozessmanagements ...................... 24

Abb. $2.4 \quad$ Strategische Ziele des Prozessmanagements - priorisiert ............. 25

Abb. 2.5 Strategische Ziele des Prozessmanagements - «1. Priorität» nach Unter-

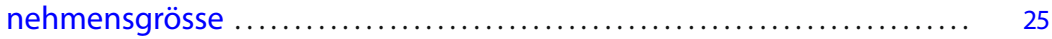

Abb. 2.6 Potenzialanalysen für die Unterstützung strategischer Entscheide .... 26

Abb. 2.7 Prozessmanagement-Mittel für strategische Entscheidungsunterstützung - «systematisch \& gelegentlich» nach Unternehmensgrösse $\ldots . . \quad 27$

Abb. $2.8 \quad$ Medienbrüche im Unternehmensalltag $\ldots \ldots \ldots \ldots \ldots \ldots \ldots \ldots \ldots \ldots . \quad 28$

Abb. 2.9 Medienbrüche im Unternehmensalltag - «Nie» nach Unternehmens-

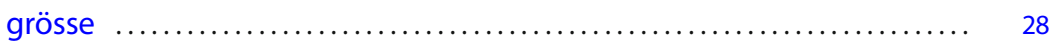

Abb. 2.10 Einsatz technischer Methoden für durchgängige Prozesse vom und

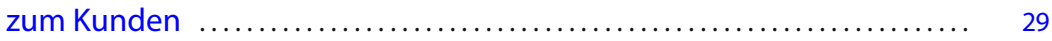

Abb. 2.11 Einsatz von Methoden für flexible und kontextsensitive Prozesse . .... 30

Abb. 2.12 Aktionsfelder der digitalen Transformation ....................... 31

Abb. 2.13 Aktionsfelder der digitalen Transformation - «umgesetzt oder angestrebt oder evaluiert» nach Unternehmensgrösse .................. 32

Abb. 2.14 Reaktionen der IT auf den unternehmerischen und technologischen

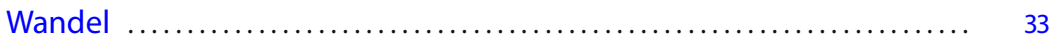

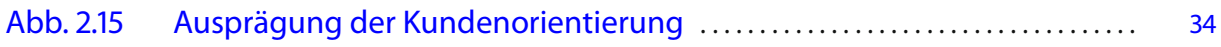

Abb. 2.16 Methoden für die Erhebung der Bedürfnisse von externen und internen Kunden ............................................... 35

Abb. 2.17 Methoden für die Erhebung der Bedürfnisse von externen und internen Kunden - «systematisch oder gelegentlich» nach Unternehmens-

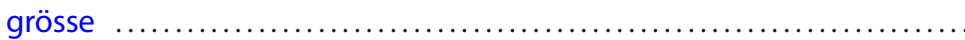

Abb. 2.18 Visualisierung von Kundeninteraktionen und -bedarf in Prozessmodellen und -landkarten ..................................... 36

Abb. 2.19 Visualisierung von Kundeninteraktionen in Prozesslandkarten ........ 37

Abb. 2.20 Visualisierung von Kundeninteraktionen in einzelnen Prozessmodellen $\quad 37$ Abb. 2.21 Nutzung der «Customer Journey«-Erkenntnisse für die kundenorientierte Prozessgestaltung/-optimierung 
Abb. 2.22 Nutzung der «Employee Journey«-Erkenntnisse für mitarbeiterorientierte Prozessgestaltung/-optimierung ........................ 38

Abb. 2.23 Aktivitäten zur Gestaltung der Kundeninteraktion .................. 39

Abb. 2.24 Aktivitäten zur Gestaltung der Kundeninteraktion - «systematisch oder punktuell» nach Unternehmensgrösse ........................ 40

Abb. 2.25 Quelle und Regelmässigkeit der Erhebung von Kundendaten .......... 41

Abb.2.26 Quelle und Regelmässigkeit der Erhebung von Kundendaten - nach Unternehmensgrösse ..................................... 42

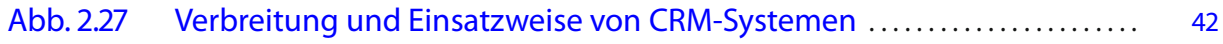

Abb. 2.28 Nutzung von Kundendaten ................................... 43

Abb. 2.29 Nutzung von Kundendaten - nach Unternehmensgrösse ............. 43

Abb. 2.30 Einflussmöglichkeiten der Kunden auf die Datenhaltung (»Unsere Kun-

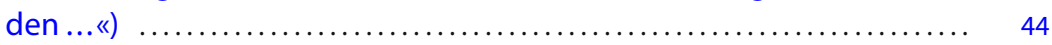

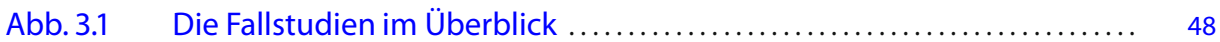

Abb. $4.1 \quad$ Online-Leasingrechner. (www.amag.ch, Stand August 2016) ........... 54

Abb. 4.2 Online-Leasingzertifikat. (www.amag.ch, Stand August 2016) .......... 55

Abb.4.3 Auswirkungen nicht GwG/GwV-FINMA-konformer Identitätsdoku-

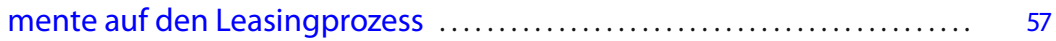

Abb. 4.4 Zielsetzung: Online-Bewilligung .............................. 59

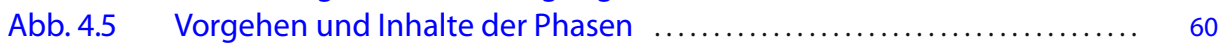

Abb. 4.6 Phase 1-Backend-Prozess und Prototyp ........................ 61

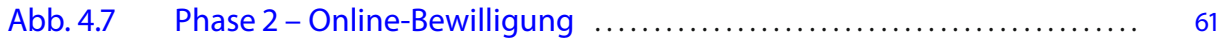

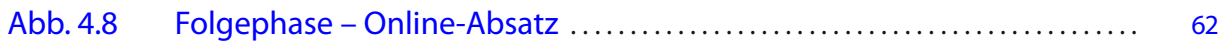

Abb. 4.9 Systemlandschaft - Status quo und Erweiterungen für das Online-Lea-

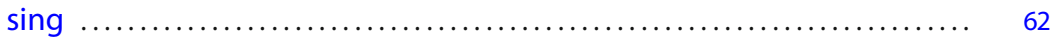

Abb. 4.10 Kooperation mit dem ZHAW Service Lab .......................... 64

Abb. 4.11 AMAG Leasing Fallstudie - Kernaspekte im Kontext des Studienframe-

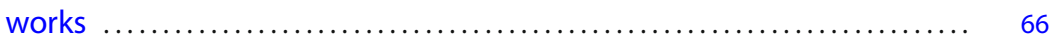

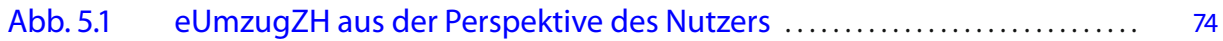

Abb. 5.2 eUmzugZH - Prozess und Lösungselemente ..................... 76

Abb. 5.3 Projektorganisation für die Umsetzung von eUmzugZH im Kontext eUmzugCH ......................................... 77

Abb.5.4 eUmzugZH Fallstudie - Kernaspekte im Kontext des Studienframeworks ................................................. 79

Abb. 6.1 Übersicht des Offertstellungsprozesses im Bereich Property \& Casualty

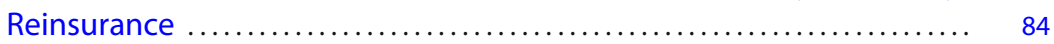

Abb. 6.2 Marktsegmente und Wachstumspotenzial ........................ 86

Abb. 6.3 Architekturübersicht des ersten Lösungsansatzes .................... 89

Abb. 6.4 Architekturübersicht des zweiten Lösungsansatzes ................. 92

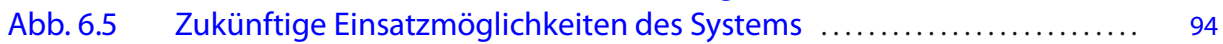

Abb. 6.6 Swiss Re Fallstudie - Kernaspekte im Kontext des Studienframeworks . 95

Abb. 7.1 Die vier Lösungselemente .................................... 102

Abb. 7.2 BPM Lifecycle in Anlehnung an. (Dumas et al. 2013) ................. 103

Abb. 7.3 Konföderierte Configuration Management Datenbank ............... 104

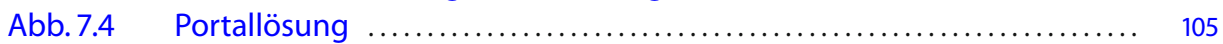

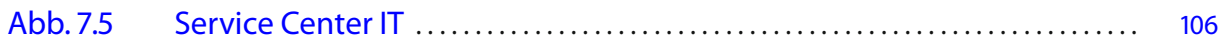

Abb.7.6 Vontobel Fallstudie - Kernaspekte im Kontext des Studienframeworks 107

Abb. $8.1 \quad$ Unternehmensstrategie «Swisscom 2020« ..................... 113 
Abb. 8.2 Rolle der Organisationseinheit «Network \& IT Operations» im Prozessmodell Swisscom ............................................. 113

Abb. 8.3 Fertigung in verteilten Fertigungsstrassen über viele OEs hinweg ..... 114

Abb. 8.4 Kernprinzipien der industriellen Fertigung, Trennung vom Was und Wie ....................................................... 115

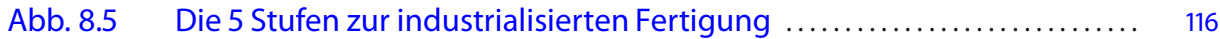

Abb. 8.6 Services-Lebenszyklus ............................................ 119

Abb. 8.7 Gesamtübersicht Ziele, Vorgehen, Lösungsansatz .................. 121

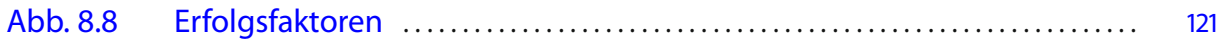

Abb. 8.9 Swisscom Fallstudie - Kernaspekte im Kontext des Studienframeworks 122

Abb. 9.1 Zusammenfassung der Ergebnisse der quantitativen Online-Befra-

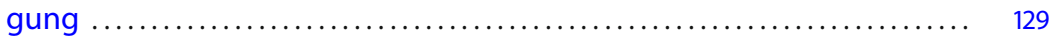

Abb. 9.2 Kundennutzen durch digitale Transformation? - Rahmenwerk der Stu-

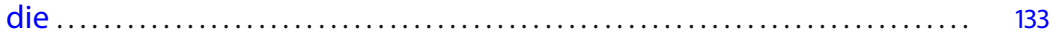

Abb. 9.3 BPM quo vadis? Entwicklungspotenziale im Kontext des digitalen Wandels ...................................................... 134

Abb. 10.1 Teilnehmende nach Land der Geschäftstätigkeit des Unternehmens .. 140

Abb. 10.2 Teilnehmende nach Grösse des Unternehmens $\ldots \ldots \ldots \ldots \ldots \ldots \ldots \ldots \ldots$

Abb. 10.3 Teilnehmende nach Land und Unternehmensgrösse ............... 141

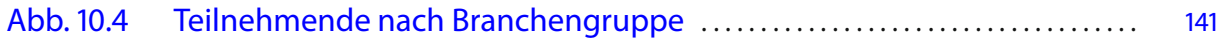

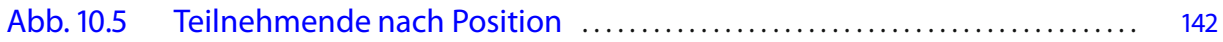

Abb. 10.6 Teilnehmende nach Funktionsbereich ........................... 142

Abb. 10.7 Teilnehmende nach Wahrnehmung einer Prozessfunktion ............. 143 


\section{Tabellenverzeichnis}

Tab. $1.1 \quad$ Elemente und Ergebnisse der Studie ........................... 11

Tab. 8.1 Eigenschaften der industriellen Fertigung f..................... 114 